\title{
Vulnerabilidad cognitiva específica de la depresión
}

\author{
Cognitive vulnerability specific to depression \\ Jennifer Verdi-Estrada ${ }^{a}$, Marlene Cañedo-Galván $^{b}$, Irma E. Andrade-Tapia $^{c}$, Gelacio \\ Guzmán-Díaz $^{d}$, Jesús Cisneros-Herrera ${ }^{e}$
}

\begin{abstract}
:
Cognitive vulnerability is a general concept used by cognitive-behavioural therapies, which is referred to mental functioning that allow for emergence of psychiatric disorders. In these therapies, thoughts are the determining factor for individual's emotions and behaviours; therefore, the origin of depression can be traced to certain kind of thoughts. In this paper, cognitive vulnerability specific to depression is addressed. This vulnerability is explained by four factors: cognitive triad, automatic negative thoughts, cognitive distortions and dysfunction in schemata related to self, the world and the future. At the end, some prevention interventions are introduced, which are based on cognitive vulnerability.
\end{abstract}

Keywords:

Cognitive vulnerability, depression, cognitive triad, prevention

Resumen:

Vulnerabilidad cognitiva es un concepto genérico adoptado por las terapias cognitivo-conductuales para referirse a las condiciones que facilitan la aparición de los trastornos psiquiátricos. En estas terapias, el pensamiento es el factor determinante de las emociones y la conducta de los individuos, por lo que el origen de los trastornos puede ubicarse en él. En este trabajo se aborda específicamente la vulnerabilidad cognitiva que se ha encontrado en la depression. Esta vulnerabilidad se explica por cuatro factoers: la triada cogniiva, pensamientos automáticos negativos, distorsiones cognitivas y disfunción en los esquemas relacionados con el sí mismo, el mundo y el futuro. Para finalizar, se presentan algunas propuestas preventivas basadas en la vulnerabilidad cognitiva.

\section{Palabras Clave:}

Vulnerabilidad cognitiva, depresión, triada cognitiva, prevención.

\section{Introducción}

El planteamiento fundamental de los modelos cognitivos es que las cogniciones median la relación entre los eventos que experimentan las personas y las emociones que sienten (1). Es decir, la manera en que la persona interpreta una situación o experiencia determina sus respuestas emocionales y conductuales. Por ejemplo, una persona que se encuentra preparando un pastel y se le quema, puede sentirse triste y pensar que no puede hacer nada bien; ya que estaría extrayendo conclusiones basándose en pocas experiencias. En cambio, si piensa que no importa, que si sigue practicando en el futuro mejorará, de esta forma sólo se sentirá tranquila y motivada por el aprendizaje. Por ello, se ha aplicado ampliamente en el tratamiento de trastornos de ansiedad y del estado de ánimo.

La teoría cognitiva considera que hay cuatro elementos cognitivos clave que explican la depresión (2): 1) la tríada cognitiva, formada por los tres esquemas o patrones

\footnotetext{
a Autor de Correspondencia, alumna de la Licenciatura en Psicología, Universidad Autónoma del Estado de Hidalgo, https://orcid.org/00000002-6295-0648, Email: ve266411@uaeh.edu.mx

b Universidad Autónoma del Estado de Hidalgo, https://orcid.org/0000-0001-6127-4089, Email: ca352840@uaeh.edu.mx c Universidad Autónoma del Estado de Hidalgo, https://orcid.org/0000-0003-0601-0923, Email: an352503@uaeh.edu.mx

d Universidad Autónoma del Estado de Hidalgo, https://orcid.org/0000-0003-4869-8658, Email: gelacio_guzman@uaeh.edu.mx e Universidad Autónoma del Estado de Hidalgo, https://orcid.org/0000-0003-1370-8086, Email: jesus_cisneros@uaeh.edu.mx
} 
cognitivos que inducen al individuo a percibirse a sí mismo, al mundo y al futuro desde un punto de vista negativo; 2) los pensamientos automáticos negativos; 3) errores en la percepción y en el procesamiento de información (distorsiones cognitivas); y 4) disfunción en los esquemas, referentes a la visión negativa sobre unos mismo, el mundo y el futuro.

Los pensamientos automáticos son los pensamientos e imágenes que resultan de la interacción de la información proporcionada por el medio, los esquemas y distorsiones en sus distintos niveles de accesibilidad (2). Por su parte, Guglielmo (3) hace mención de que un pensamiento automático negativo es aquella cognición involuntaria, ya sea verbal, subverbal o no verbal compuesta de imágenes experimentadas por individuos, y que puede ser clínicamente relevante si se determina que influye y/o se asocia con síntomas o comportamientos negativos. Los pensamientos automáticos pueden representar ideas y creencias superficiales, o lo que se ha llamado creencias centrales. Es decir, son todos aquellos productos cognitivos, o bien, las ideas involuntarias que surgen bajo la correlación de los procesos cognitivos y los esquemas. El contenido de estos productos cognitivos suele ser de más fácil acceso. Una forma de ejemplificar los pensamientos automáticos sería: cuando un alumno tiene una tarea académica por entregar y la cual le resulta muy difícil de realizar, lo primero que piensa ante esta situación es: "Es mucho trabajo. Soy muy malo en esta materia. No creo que pueda hacerlo. Voy a reprobar."

Por un lado, Guglielmo (3) define una distorsión cognitiva como la presencia de contenido de pensamiento idiosincrásico, indicativo de conceptualizaciones distorsionadas o poco realistas. Por otro lado, Ruiz, Díaz y Villalobos (2) mencionan que las distorsiones cognitivas son las reglas transformacionales a través de las cuales los individuos seleccionan del medio la información que será atendida, codificada, almacenada y recuperada. Cuando los sujetos se enfrentan a una situación estimular determinada, no analizan toda la información disponible, sino que atienden a indicios que ya están contenidos o son congruentes con la información de esquemas cognitivos preexistentes. Por lo tanto, ante determinadas situaciones las personas no suelen responder de manera automática, sino que antes de emitir una respuesta, tanto emocional como conductual, las personas tienden a percibir, evaluar, clasificar, interpretar y asignar un significado a dicha situación en función a una manera de respuesta. Continuando con el ejemplo anterior (tarea académica), los procesos cognitivos que se presentan ante esta situación son: etiquetas globales, minimización y catastrofismo.

Los esquemas son entidades organizativas conceptuales complejas compuestas de unidades, y contienen conjuntos de creencias nucleares relacionadas con la visión del mundo, de los otros y sobre uno mismo y su interacción con los demás, estos esquemas son internos (2). Asimismo, Guglielmo (3) menciona que los esquemas se definen como una estructura cognitiva interna compuesta de componentes epistémicos (recuerdos de estímulos, componentes actitudinales, creencias) que influyen y/o guían el procesamiento y el comportamiento de la información humana. Es decir, son estructuras cognitivas relativamente permanentes organizadas a través de la experiencia previa y que permiten percibir, codificar y evaluar el rango total de estimulación interna y externa, así como decidir el curso de acción subsiguiente. Continuando con el ejemplo mencionado en los párrafos anteriores, los esquemas que se hacen presentes, serían los siguientes: Fracaso y negativismo / pesimismo.

\section{Trastornos depresivos}

La manera en que las personas interpretan eventos particulares aumenta el riesgo de desarrollar trastornos emocionales en el futuro. Puede incluir fenómenos distales que estaban presentes antes del trastorno, y fenómenos proximales que ocurren muy cerca o incluso durante el evento. Los factores cognitivos distales son normalmente predisposiciones cognitivas relativamente duraderas, predisposiciones para responder a situaciones estresantes de manera desadaptativa $(1,4,5)$.

La vulnerabilidad cognitiva hace referencia a las creencias defectuosas, sesgos cognitivos o estructuras que se hipotetizan para establecer el escenario de problemas posteriores (5). Se ha encontrado que, en distintos trastornos psicológicos, se presenta un patrón diferente de tipo de vulnerabilidad cognitiva, por lo que se llegó a la conclusión que ciertos patrones propician la aparición de los trastornos. Por ejemplo, en el trastorno paranoide de la personalidad, la vulnerabilidad que este trastorno presenta es la siguiente: 1) Triada cognitiva: a) Percepción de sí mismo: Inocente, noble, vulnerable. b) Percepción del mundo: Maliciosos, interferentes y discriminadores 2) Pensamientos automáticos: "Soy vulnerable a otras personas", "si no tengo cuidado me manipularán, abusarán o se aprovecharán de mí", "si la gente actúa amistosamente, es porque tratan de usarme", "los demás me engañarán debido a sus malas intenciones", "me van a desacreditar-derrumbar", "no se puede confiar en los demás"; 3) Distorsiones cognitivas: Sobregeneralización, pensamiento todo o nada (dicotómico) y lectura de pensamiento (adivinación) (6); 4) Esquemas: Desconfianza / abuso, privación emocional y aislamiento social (7).

En los trastornos depresivos, el rasgo común de estos es la presencia de un ánimo triste, vacío o irritable, acompañado de cambios somáticos y cognitivos que 
afectan significativamente a la capacidad funcional del individuo (8).

La vulnerabilidad dentro de los trastornos depresivos a menudo refleja pérdida, deprivación, inutilidad o derrota, lo cual se explica a través de la triada cognitiva $(2,9)$ : a) la visión negativa de sí mismo: el paciente se ve desgraciado, torpe, enfermo, con poca valía, por lo que tiende a atribuir sus experiencias desagradables a un defecto suyo, de tipo psíquico, moral, o físico. Debido a esto, el paciente cree que, a causa de estos defectos, es un inútil y carece de valor; b) la visión negativa del mudo: se centra en la tendencia del depresivo a interpretar sus experiencias de una manera negativa, le parece que el mundo le hace demandas exageradas y/o le presenta obstáculos insuperables para alcanzar sus objetivos; c) la visión negativa acerca del futuro: cuando la persona depresiva hace proyectos de gran alcance, está anticipando que sus dificultades o sufrimientos actuales continuarán indefinidamente, por lo que espera penas, frustraciones y privaciones interminables.

Los patrones cognitivos negativos causarán el resto de los signos y síntomas del síndrome depresivo. Por ejemplo, si el paciente piensa anticipadamente que va a ser rechazado, reaccionará con el mismo efecto negativo (tristeza, enfado) que cuando el rechazo es real. Si piensa erróneamente que vive marginado de la sociedad, se sentirá solo.

Los esquemas disfuncionales relacionados con la patología depresiva se caracterizarían por ser rígidos, impermeables y concretos, frente a los más flexibles y adaptativos propios de la normalidad (10). De acuerdo con Dowd (11), los esquemas maladaptativos que pueden estar asociados a la depresión son los de deprivación emocional, vergüenza/defectuosidad y fracaso.

De acuerdo con los primeros escritos de Beck, se identifican 6 distorsiones que cometían los pacientes depresivos: inferencia arbitraria ("no voy a encontrar trabajo nunca, no valgo para nada"), abstracción selectiva ("tengo mucho tiempo sin ilusión por nada, creo que nunca volveré a recuperar la ilusión por las cosas como hacía antes"), generalización excesiva ("solamente me pasan cosas malas"), maximización/minimización ("no me importan mis éxitos pasados, lo que ahora importa es que he cometido ese grave error"), personalización ("últimamente me digo a mí misma que todo es culpa mía por ser como soy") y pensamiento absolutista / dicotómico ("si no acredito la materia soy un incompetente e inútil) (2, $3,9)$.

Los pensamientos poseen informacion proposicional que es única para el individuo y como consecuencia, no está integrado en lo que se considera creencia compartida. La "validez", si se toma al pie de la letra, comunica una naturaleza ilógica a la distorsión cognitiva ya que la validez se refiere a la naturaleza conservadora de la verdad de una inferencia (3). Los pensamientos automáticos estarían determinados por los esquemas cognitivos más profundos, es decir, cada pensamiento es individualizado, sin embargo se encuentran englobados dentro de los pensamientos de autoconcepto negativo, de dificultad de afrontamiento y de insatisfacción (12).

\section{Intervenciones preventivas}

Londoño (13) hace mención de la intervención preventiva de la vulnerabilidad cognitiva de la depresión, abarcando la fase primaria, secundaria y terciaria.

\section{Prevención primaria:}

1. Identificar jóvenes con riesgos de depresión.

2. Se interviene de manera grupal el afrontamiento al estrés, la regulación emocional, el proceso de enamoramiento y su incidencia en la depresión en la distinta.

3. Incidencia de padres depresivos.

4. Hablar sobre la importancia de este trastorno, la incidencia que tiene los problemas en el estado de ánimo, la herencia y los antecedentes depresivos en los padres, episodios depresivos pasados, ser mujer, y los beneficios de recibir apoyo terapéutico.

5. Las técnicas cognitivo conductuales orientadas a lo modificación de creencias negativas con relación a sí mismos y a los demás, a la modificación de esquemas maladaptativos y al cuestionamiento de ideas irracionales e intervenidas de manera individual y grupal para solucionar dificultades de la vida personal, de pareja y familiar (restructuración cognitiva).

6. Técnicas para identificar y diferenciar los pensamientos y las emociones, y la funcionalidad de las emociones.

7. La terapia en autocontrol para estimular procesos de auto-observación, autoevaluación y autoesfuerzo.

8. Programación de eventos positivos como facilitador de los estados de bienestar y atención a las experiencias positivas.

9. Desarrollo en habilidades sociales. Técnicas en solución de problemas.

10. Técnicas en relajación.

Prevención secundaria

Identificación de factores de riesgo para prevenir el desarrollo del trastorno:

11. Predictores Sociodemográfico.

12. Predictores psicológicos.

13. Estresores psicosociales y ambientales. 
14. Predictores clínicos y neuropsiquiátricos.

Prevención terciaria

Se utiliza el modelo de la terapia cognitiva, en donde la función del terapeuta es ayudar a que el paciente reorganice su estilo de pensamiento y utilice la evidencia, el pensamiento lógico y la razón para analizar las distorsiones que provocan alteraciones emocionales. En el enfoque terapéutico, el terapeuta se dirige hacia las distorsiones cognitivas para obtener valoraciones mucho más correctas de la realidad, que se reflejan en reducciones significativas de la depresión.

Por su parte, Londoño, Jaramillo, Castaño, Rivera, Berrio y Correa (14), en su estudio, utilizaron un diseño cuasiexperimental, evaluación pre-test y pos-test y grupo control. Participaron 60 estudiantes de dos universidades de Colombia que cursaban su primer año de formación, con una edad promedio de 18.5; los grupos se conformaban por: Grupo experimental $\mathrm{N}=31$ y grupo control $\mathrm{N}=29$. El programa de prevención se realizó con la modalidad de taller y se centró en la prevención de la depresión y la ansiedad a través del modelo del desarrollo del pensamiento crítico y la pregunta socrática, el tiempo programado fue de 5 sesiones de 2 h cada una. Se identificaron cambios significativos en los indicadores clínicos de depresión, ansiedad, pensamientos negativos y valoración de acontecimientos vitales en el grupo experimental. El programa de prevención de la depresión y la ansiedad a través del modelo del desarrollo del pensamiento crítico y el diálogo socrático, es efectivo en jóvenes universitarios del primer año de formación, en la medida que disminuye significativamente los indicadores de depresión y ansiedad, los pensamientos negativos y la valoración de los acontecimientos vitales.

Por otra parte, Londoño, Palacio, Acosta, Juárez y Aguirre (15) en su estudio participaron 110 universitarios que accedían al primer año de la carrera; se utilizó un diseño experimental aleatorizado con grupo experimental y grupo control. Al comparar los resultados entre los dos grupos, se observó que el programa resultó efectivo en la reducción de los pensamientos negativos para el grupo experimental, aunque no se identificaron otros efectos. El diálogo socrático y el pensamiento crítico en el marco de un programa de prevención, reducen los pensamientos negativos en los universitarios de primer año.

\section{Conclusiones}

El modelo cognitivo se basa en la medición de las cogniciones, abarcando elementos como los pensamientos automáticos, las distorsiones cognitivas, los esquemas y la triada cognitiva. Por lo tanto, estos elementos influirán en la manera en que las personas interpretan eventos particulares y las emociones que sienten, aumentando el riesgo de desarrollar trastornos emocionales en el futuro. Los sesgos cognitivos que producen dichos elementos constituyen la vulnerabilidad cognitiva.

La vulnerabilidad cognitiva en los trastornos depresivos a menudo refleja pérdida, deprivación, inutilidad o derrota. Por lo tanto, es importante el desarrollo e implementación de programas preventivos dirigidos hacia la vulnerabilidad cognitiva específica de la depresión, para así poder lograr un declive en la tasa de prevalencia de los trastornos depresivos. Si bien es cierto que, los estudios encontrados acerca de la prevención de la vulnerabilidad cognitiva específica de la depresión han sido pocos, no quiere decir que no sean favorables, ya que en los estudios citados en la presente investigación han mostrado resultados favorables para sus muestras.

\section{Referencias}

[1] Alloy L, Riskind J. Cognitive vulnerability to emotional disorders. Newjersey: Laurens Erlbauna Association Publisher; 2006.

[2] Ruiz M, Diaz M, Villalobos A. Manual de técnicas de intervención cognitivo conductual. Bilbao, España: Desclée Brouwer; 2012.

[3] Guglielmo S. Cognitive distortion: propositions and possibleworlds. Journal of Rational-Emotive \& Coognitive-behavior therapy 2014; 33(1): 53-77.

[4] Carrasco A. Modelos psicoterapéuticos para la depresión: hacia un enfoque integrado. Intramerican Journal of Psychology 2017; 51(2): 181-189.

[5] Riskind J. Black D. Cognitive Vulnerability. En A. Freeman, S. Felgoise, C. Nezu, A. Nezu, \& M. Reinecke, Encyclopedia of congnitive behavior therapy. Boston: Springer; 2005: 122-126.

[6] López A, Rondón J, Cellerino C, Alfano S. Guías esquematizadas de tratamiento de los trastornos de la personalidad para profesionales, desde el modelo de Beck, Freeman, Davis y otros (2005). Ciencias Psicológicas 2010; 4(1): 97-124.

[7] López A, Colom C, Camerini O, Rondón J, Cellerino C, Alfano S. Guías esquematizadas de tratamiento de los trastornos de la personalidad para profesionales, desde el modelo de Young, Klosko y Wheishar (2003). Ciencias Psicológicas 2011; 5(1): 83-115.

[8] Asociación Americana de Psiquiatría. Manual diagnóstico y estadístico de los trastornos mentales. 5a ed. Buenos Aires, Bogotá, Caracas, Madrid, México, Porto Alegre: Médica Panamericana; 2014.

[9] Beck A, Rush A, Shaw B, Emery G. Terapia cognitiva de la depresión. España: Desclée De Brouwer; 2012.

[10] Hernangómez L. Vulnerabilidad cognitiva a la depresión: relación entre sesgos atencionales, auto-representación y síntomas depresivos. Tesis doctoral, Universidad complutense de Madrid, Departamento de Personalidad, Evaluación y Tratamiento Psicológico I, Madrid; 2012. 
[11] Dowd T. Depression: Theory, asessment, and new directions in practice. International Journal of Clinical and Heatlh Psychology 2004 ; 4(2): 413-423.

[12] Estévez A. Calvete E. Mediación a través de pensamientos automáticos de la relación entre esquemas y síntomas de depresión. Anales de Psicología 2009; 25(1): 27-35.

[13] Londoño N. Predictores cognitivos, de personalidad y estres para sintomas de depresión y ansiedad en estudiates de primeros semestres de educación superior e inpactodel programa de prevención basado en el desarrollo del pensamiento crítico y el diálogo socrático. Tesis doctoral, Universidad del Norte; 2011.

[14] Londoño N, Jaramillo J, Castaño M, Rivera D, Berrio Z, Correa D. Prevención de la depresión y la ansiedad en estudiantes universitarios. Psicologia e Saúde 2015; 7(1): 47-55.

[15] Londoño N, Palacio J, Acosta C, Juárez F, Aguirre D. Efectividad de un programa de prevención ensalud mental mediante el diálogo socrático y el pensamiento crítico. Salud Uninorte 2016; 32(1): 1-24. 